\title{
The Adductor Strengthening Programme prevents groin problems among male football players: a cluster-randomised controlled trial
}

\author{
Joar Harøy, ${ }^{1}$ Benjamin Clarsen, ${ }^{1}$ Espen Guldahl Wiger, ${ }^{1}$ Mari Glomnes Øyen, ${ }^{1}$ \\ Andreas Serner, ${ }^{2,3}$ Kristian Thorborg, ${ }^{2}$ Per Hölmich, ${ }^{2}$ Thor Einar Andersen, ${ }^{1}$ Roald Bahr ${ }^{1}$
}

\begin{abstract}
- Additional material is published online only. To view please visit the journal online (http://dx.doi.org/10.1136/ bjsports-2017-098937).

'Department of Sports Medicine, Norwegian School of Sport Sciences, Oslo Sports Trauma Research Center, Oslo, Norway ${ }^{2}$ Department of Orthopedic Surgery, Sports Orthopedic Research Center, Copenhagen, Copenhagen University Hospital, Amager-Hvidovre, Denmark ${ }^{3}$ Aspetar Orthopaedic and Sports Medicine Hospital, Doha, Qatar
\end{abstract}

\section{Correspondence to}

Joar Harøy, Department of Sports Medicine, Norwegian School of Sport Sciences, Oslo Sports Trauma Research Center, Oslo 0806, Norway; joar.haroy@nih.no

Received 12 December 2017 Revised 9 April 2018 Accepted 17 May 2018 Published Online First 10 June 2018

\section{ABSTRACT}

Background Groin injuries represent a considerable problem in male football. Previous groin-specific prevention programmes have not shown a significant reduction in groin injury rates. An exercise programme using the Copenhagen Adduction exercise increases hip adduction strength, a key risk factor for groin injuries. However, its preventive effect is yet to be tested.

Aim To evaluate the effect of a single-exercise approach, based on the Copenhagen Adduction exercise, on the prevalence of groin problems in male football players.

Methods 35 semiprofessional Norwegian football teams were cluster-randomised into an intervention group (18 teams, 339 players) and a control group (17 teams, 313 players). The intervention group performed an Adductor Strengthening Programme using one exercise, with three progression levels, three times per week during the preseason (6-8 weeks), and once per week during the competitive season ( 28 weeks). The control group were instructed to train as normal. The prevalence of groin problems was measured weekly in both groups during the competitive season using the Oslo Sports Trauma Research Center Overuse Injury Questionnaire. Results The average prevalence of groin problems during the season was $13.5 \%(95 \% \mathrm{Cl} 12.3 \%$ to $14.7 \%)$ in the intervention group and $21.3 \%(95 \%$ $\mathrm{Cl} 20.0 \%$ to $22.6 \%$ ) in the control group. The risk of reporting groin problems was $41 \%$ lower in the intervention group $(\mathrm{OR} 0.59,95 \% \mathrm{Cl} 0.40$ to 0.86 , $\mathrm{p}=0.008$ ).

Conclusion The simple Adductor Strengthening Programme substantially reduced the self-reported prevalence and risk of groin problems in male football players.

Trial registration number ISRCTN98514933.

\section{INTRODUCTION}

Groin injuries represent a considerable problem in male football, accounting for 4\%-19\% of all timeloss injuries. ${ }^{1}$ At the elite level, approximately one in five male players incur a groin injury causing time loss each season. ${ }^{2}$ To date, the effect of groin-specific prevention programmes has not been established. ${ }^{3} \mathrm{~A}$ recent systematic review and meta-analysis indicates that the FIFA $11+$ mayhave a preventive effect on hip and groin injuries. ${ }^{4}$ However, this analysis was based on two studies only. A significant reduction in groin injury rate was observed among collegiate male players, ${ }^{5}$ while there was no difference in a study on young female players. ${ }^{6}$
One limitation of previous groin-specific prevention studies is the use of a time-loss injury definition, an inadequate approach as only about one-third of all groin problems result in time loss. ${ }^{7}$ Injuries causing time loss may only represent the 'tip of the iceberg' as a large proportion of players continue to participate despite having groin-related complaints with associated impairments or reduced performance. ${ }^{17-9}$ To address this, we have developed a new method to improve the recording of groin problems, using an 'all physical complaints' approach, in order to capture all cases leading to pain, decreased participation or performance, not only those resulting in time loss. ${ }^{10}$

In football, two out of three cases of groin injuries are adductor-related. ${ }^{211-14}$ Low hip adduction strength has been identified as an important and modifiable risk factor associated with an increased risk of groin injury. ${ }^{15} 16$ Furthermore, $>20 \%$ deficit in eccentric strength of the hip adductor muscles has been observed among players with groin pain. ${ }^{17}$ Thus, strengthening the hip adductors may play an important role in reducing the prevalence and rate of groin injuries in football players. In recent studies, the muscle activation patterns and strength effects of various hip adductor exercises have been examined. ${ }^{18-21}$ The Copenhagen Adduction exercise (CA) has demonstrated high activation of the adductor longus muscle, ${ }^{21}$ as well as considerable eccentric adduction strength gains following standardised protocols. ${ }^{18} 19$ The apparent advantage of this exercise is that no equipment is needed and the CA can be performed on the pitch just prior to or after training. However, its preventive effect has not been tested.

Thus, the purpose of this cluster-randomised controlled trial (RCT) was to test the effect of a single-exercise approach, based on the CA, to reduce the prevalence of groin problems in male players.

\section{METHODS}

\section{Study design and participants}

This cluster-RCT was registered with the International Standard Randomised Controlled Trial Number registry (ISRCTN98514933) and took place from February 2016 to October 2016. During the 2016 preseason (February and March), we invited teams from the second and third levels (semiprofessional) in Norway to take part in the study. We visited all teams who agreed to participate to inform and invite players to participate. All players under contract with the team were eligible for participation, except those not expected to train 
or play during the first $6-8$ weeks of the season due to injury or illness. The players received verbal and written information about the study purpose and procedures and provided written consent to participation. All players included at baseline were followed until the end of the season (October).

This report is prepared according to the TIDieR checklist and the Consort Statement recommendations with extension for reporting cluster-randomised trials. ${ }^{22} 23$

\section{Baseline questionnaires}

We registered demographic data, playing position, dominant leg (kicking leg) and years as a senior player. Players also registered current hip and groin symptoms using the Oslo Sports Trauma Research Center (OSTRC) Overuse Injury Questionnaire ${ }^{10}$ and the Copenhagen Hip and Groin Outcome Score (HAGOS) at baseline. $^{24}$

\section{Randomisation}

We cluster-randomised on a team level to minimise the risk of contamination bias between players in the intervention and control groups. A statistician, who was blinded to the study protocol, performed a computer-generated block randomisation, with block sizes of 4 and 6 in random order. After a team agreed to participate, the principal investigator opened a sealed envelope revealing their group assignment.

\section{Blinding}

It was not possible to blind players, coaches or the principal investigator to group allocation. However, a research assistant was blinded and managed all data during the collection period, and outcome measures were not available to any party until all data had been collected.

\section{Intervention}

The Adductor Strengthening Programme consisted of a single exercise with multiple levels of difficulty. The exercise was based on the CA as this has previously been shown to be a high-intensity exercise targeting the adductors. ${ }^{21}$ As the CA might be painful or difficult to perform for symptomatic players, we created two easier levels that players could choose from: level 1 (easiest): side-lying hip adduction; level 2 (moderate): the CA as previously described, ${ }^{21}$ but with a shorter lever arm; and level 3 (hardest): the CA as previously described ${ }^{21}$ (figure 1). Videos with detailed information on the performance of each level are available as an online supplementary appendix .

Players were asked to start at level 3. However, if they experienced groin pain during the exercise $>3$ on an 11-point numeric rating scale $\left(0-10\right.$, where 0 is no pain and 10 is maximal pain), ${ }^{25}$ they were instructed to perform level 2 instead. Similarly, if level 2 provoked pain $>3 / 10$, the player was informed to perform level 1. The exercise was performed on both sides.

Teams in the intervention group were asked to perform the programme as a part of their regular warm-up, 2-3 times a week for a minimum of 6 weeks during the preseason and maintain the programme once a week throughout the regular season (table 1). At a team visit during preseason, players and coaches in the intervention group were shown how to perform the different levels of the programme by the principal investigator. Team medical staff were also included in the instruction session whenever
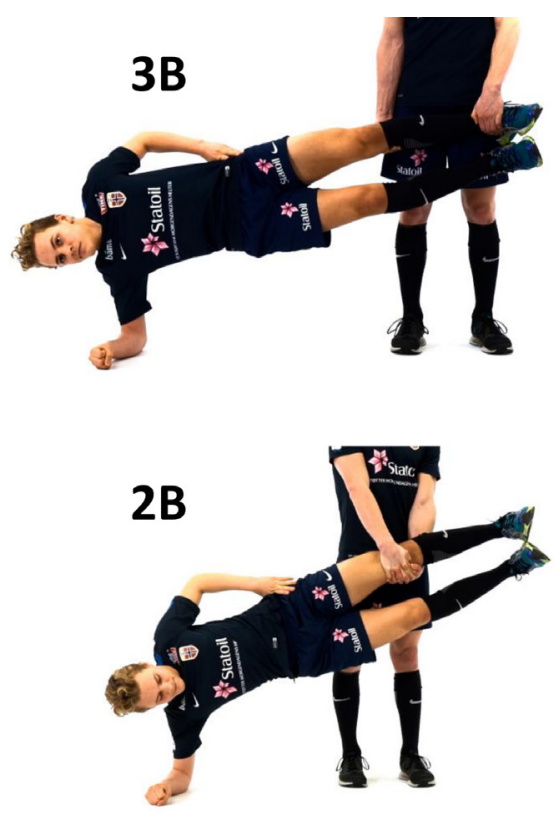

1B

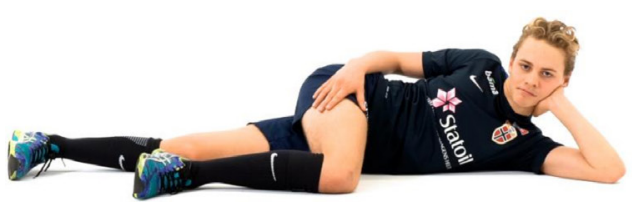

Figure 1 (A) Starting/ending position and (B) mid position for the different levels of the Adductor Strengthening Programme. 


\begin{tabular}{|c|c|c|c|}
\hline Week & Weekly sessions & Sets per side & Repetitions per side \\
\hline \multicolumn{4}{|c|}{ Preseason (weeks) } \\
\hline 1 & 2 & 1 & $3-5$ \\
\hline 2 & 3 & 1 & $3-5$ \\
\hline $3-4$ & 3 & 1 & $7-10$ \\
\hline $5-6$ & 3 & 1 & $12-15$ \\
\hline $7-8$ & 2 & 1 & $12-15$ \\
\hline In season & 1 & 1 & $12-15$ \\
\hline
\end{tabular}

possible ( $75 \%$ of the time). We encouraged the players, coaches and medical staff to contact us if they experienced problems or any other adverse events when performing the exercise.

\section{Compliance}

Compliance to the training programme was based on players' self-reported number of sessions completed. This information was collected weekly as a part of an electronic questionnaire, which also measured groin injury consequences (see below). To calculate the overall compliance, the total number of completed sessions was summed and divided by the number of respondents. However, to avoid overestimation, players who performed more than the recommended number of sessions were assigned the maximum recommended number.

\section{Outcome measures}

The primary outcome measure was the weekly prevalence of all groin problems registered during the competitive season (28 weeks) using the OSTRC Overuse Injury Questionnaire. ${ }^{10}$ The secondary outcome measure was the weekly prevalence of substantial groin problems registered during the competitive season. Players were categorised as having a groin problem if they recorded any hip or groin symptoms in the questionnaire, including 'pain, ache, stiffness, clicking/catching or other complaints related to the groin', or if they reported reduced training participation, training volume or performance due to groin problems. ${ }^{10}$ Players were categorised as having a substantial groin problem if they reported moderate or severe reductions in training volume or football performance, or a complete inability to participate due to groin problems. ${ }^{10}$ The complete questionnaire is available as an online supplementary appendix.

The questionnaire was distributed using a smartphone application (Spartanova; Spartanova NV, Ghent, Belgium) during the competitive season. Each player received a notification through the smartphone application to complete the questionnaire every Sunday evening and a short message service (SMS) notification every Monday. Non-responders received an SMS reminder the following Thursday. Players who failed to respond through the smartphone application were contacted by telephone and asked to complete the questionnaire verbally. During the last fourweeks of the season (September/October), we visited each club and asked players to respond to the questionnaire retrospectively for weeks with missing responses from the start of the competitive season to the beginning of September. To assist recall, each player was shown a document summarising all their questionnaire responses and match fixtures. The retrospective registration represents a deviation from the original protocol.

Each week, we calculated the prevalence of all groin problems and substantial groin problems by dividing the number of reported problems by the number of questionnaire respondents. ${ }^{10}$
At the end of the season, the average prevalence of all problems and substantial problems was calculated, as well as a $95 \%$ CI. Finally, we calculated the cumulative incidence for all groin problems and substantial groin problems as the number of new cases each during the study period divided by the number of players included in the intention-to-treat (ITT) analysis.

\section{Exposure}

Each week, players reported their exposure to football training (hours), individual training (hours), match play (minutes) and the number of games in which they had participated. The average weekly exposure was calculated for each measure for both groups.

\section{Sample size}

The sample size calculation was based on the average prevalence of all groin problems among male Norwegian football players $(29 \%) .^{7}$ Based on an analysis of variance of within-subject and within-team prevalence, an inflation factor of 1.65 , a cluster size of 20 players, a power of $80 \%$ and a $5 \%$ significance level $(\alpha)$, we estimated the ability to detect a $40 \%$ reduction in prevalence with 380 players (19 teams) in each group.

\section{Statistical analyses}

To assess the effect of the intervention, we performed two generalised estimating equation (GEE) analyses, one for all groin problems and one for substantial groin problems. GEE was performed using an exchangeable covariance matrix and an $\alpha$ of 0.05 for all analyses. All anthropometric and demographic variables were treated as potential effect modifiers and included as candidate variables in a final multivariable GEE model built using a forward selection procedure. Analyses were performed for the 28-week period during the competitive season (April-October).

Missing data on groin problems were imputed using multivariate imputation by a chained equation algorithm in combination with a predictive mean matching approach, which led to the pooled results of five multiple imputed data sets. ${ }^{26}$ In order to perform imputation with sufficient data, we chose to remove players having $<75 \%$ response rate (equivalent to $<21$ weekly reports). The cut-off was based on an assessment of the distribution of total responses, which showed a distinct cut-off at 21 reports. This decision represents a deviation from the original protocol; however, it was made prior to performing the GEE analyses.

Primary analyses were performed using an ITT principle. We also performed secondary per-protocol (PP) analyses, in which we excluded players who performed $<67 \%$ of the programme during the preseason or $<50 \%$ during the competitive season.

We assessed between-group differences in baseline characteristics and exposure using an independent t-test or Mann-Whitney $\mathrm{U}$ test, as appropriate. We also assessed differences in baseline characteristics between players included in the ITT analysis and those excluded due to an insufficient response rate $(<75 \%)$. All analyses were performed using SPSS Statistics for Windows, V.24.0 (SPSS).

\section{RESULTS}

\section{Participants}

A total of 35 teams (652 players) were enrolled in the study; however, one team withdrew shortly after randomisation. The flow of the teams/players is shown in figure 2. Baseline characteristics for players included in the ITT analysis are shown in table 2. At the time of inclusion, there were no group 


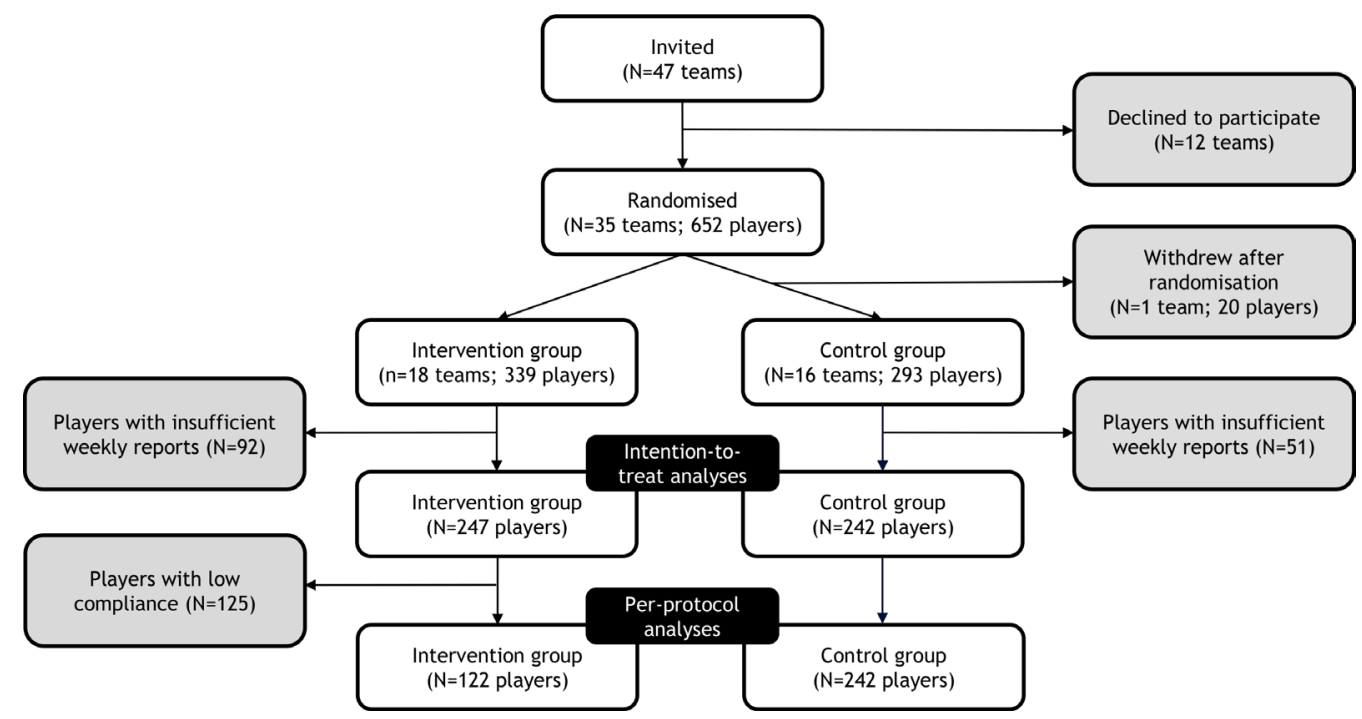

Figure 2 Flow of the teams and players throughout the intervention.

differences in the prevalence of groin problems or any of the HAGOS subscale scores. Players included in the ITT analysis did not differ from the players not meeting the $75 \%$ response rate criteria in any baseline characteristic or the prevalence of groin problems. However, the excluded players reported lower scores in three subscales of the HAGOS: sport and recreational activities (median 87.5 points vs 93.8 points), participation in physical activity (87.5 vs 100.0$)$ and quality of living (85.0 vs 90.0) at baseline. There were no differences in any of the subscales when comparing excluded players from the intervention group with the ones excluded from the control group.
There were no differences in exposure to football training, individual training, match minutes or number of games played during the competitive season between the intervention and control groups (table 3).

\section{Response rate to the weekly questionnaire}

A total of 13628 weekly reports were registered, of which $17 \%$ were registered retrospectively. The average weekly response rate was $74 \%$ (range $47 \%-93 \%$ ) in the intervention group and $80 \%(52 \%-96 \%)$ in the control group. Seventy-seven per cent

Table 2 Baseline characteristics for players included in the intention-to-treat analysis



*Values expressed as mean (SD).

tIntervention group ( $n=225)$, control group $(n=216)$.

$\ddagger$ Values expressed as median (IQR). 
Table 3 Average weekly exposure for players included in the intention-to-treat analysis, shown as the mean (SD)

\begin{tabular}{lclc}
\hline & Intervention $(\mathbf{n}=247)$ & Control $(\mathbf{n}=242)$ & P values \\
\hline $\begin{array}{l}\text { Football training (hours) } \\
\text { Individual training }\end{array}$ & $6.0(2.0)$ & $6.2(2.2)$ & 0.23 \\
$\begin{array}{l}\text { (hours) } \\
\text { Match minutes }\end{array}$ & $3.5(2.4)$ & $3.6(2.3)$ & 0.82 \\
Number of games & $05.7(26.9)$ & $67.0(25.5)$ & 0.60 \\
\hline
\end{tabular}

of players included at baseline responded to at least $75 \%$ of the weekly questionnaires and were included in the ITT analyses. The average weekly response rate for players included in the ITT analyses was 90\% (weekly range 62\%-100\%) in the intervention group and 90\% (61\%-100\%) in the control group. Furthermore, 21\% retrospective data was included in the ITT analyses and 10\% missing data were imputed using multivariate imputation.

Primary outcome: all groin problems

The average weekly prevalence of all groin problems during the competitive season was $13.5 \%$ (95\% CI $12.3 \%$ to $14.7 \%)$ in the intervention group and $21.3 \%$ (95\% CI $20.0 \%$ to $22.6 \%$ ) in the control group. The 28 in-season prevalence measures in both groups are illustrated in figure 3. GEE analyses revealed a $41 \%$ lower risk of reporting groin problems in the intervention group compared with the control group (table 4).

\section{Secondary outcome: substantial groin problems}

The average weekly prevalence of substantial groin problems during the competitive season was 5.7\% (95\% CI 5.1\% to 6.3\%) in the intervention group and $8.0 \%$ (95\% CI $7.5 \%$ to $8.5 \%)$ in the control group. The 28 in-season prevalence measures in both groups are illustrated in figure 3. An 18\% lower risk of reporting substantial groin problems was detected; however, this was not significant (table 4).

\section{Per protocol analyses: all and substantial groin problems}

When we removed the players not meeting the PP criteria ( $\geq 67 \%$ of the programme during the preseason and $\geq 50 \%$ during the competitive season), the average weekly prevalence of groin problems in the intervention group was $11.7 \%$ (95\% CI $10.9 \%$ to $12.5 \%$ ) while that of substantial groin problems was $4.5 \%$ (95\% CI $4.1 \%$ to $5.1 \%$ ). Results from GEE analysis are shown in table 4.


Figure 3 Prevalence of all groin problems (upper panel) and substantial groin problems (lower panel) in the intervention group (open squares) and control group (filled squares) with $95 \% \mathrm{Cl}$, measured at baseline and 28 times during competitive season for players included in the intention-to-treat analyses. The shaded area represents the period 6-8 weeks the preseason protocol of the Adductor Strengthening Programme was performed. 
Table 4 Generalised estimating equation (GEE) model for both intention-to-treat and per-protocol analyses.

\begin{tabular}{lllll}
\hline & $\begin{array}{c}\text { Mean difference } \\
\text { in prevalence (\%) }\end{array}$ & $\mathbf{O R}^{*}$ & $\mathbf{9 5 \% \mathrm { Cl }}$ & P values \\
\hline All problems & & & & \\
$\quad$ Intention-to-treat & 7.8 & 0.59 & 0.40 to 0.86 & 0.008 \\
\hline $\begin{array}{c}\text { Per-protocol } \\
\text { Substantial problems }\end{array}$ & 9.6 & 0.53 & 0.36 to 0.78 & 0.001 \\
\hline Intention-to-treatt & 2.3 & & & \\
\hline Per-protocol $\neq$ & 3.4 & 0.82 & 0.51 to 1.33 & 0.42 \\
\hline
\end{tabular}

${ }^{*}$ All analyses performed using intervention group as reference value.

tAge, years as senior, height and weight were included as covariates in the GEE model.

¥Height was included as a covariate in the GEE model.

\section{Characteristics of the reported groin problems}

Groin problems were reported in 2458 of the 13628 questionnaire responses. The cumulative incidence of all groin problems for players included in the ITT analysis was $55 \%$ in the intervention group and $67 \%$ in the control group. A substantial groin problem was reported by $28 \%$ of the players in the intervention group and $37 \%$ in the control group. Characteristics of the reported problems are shown in table 5 .

\section{Compliance to the exercise programme}

Players in the intervention group included in the ITT analysis completed on average $73 \%$ of the recommended preseason protocol. During the competitive season, the Adductor Strengthening Programme was completed on average 0.7 times per week (range 0.6-0.9), equivalent to $70 \%$ of the recommended exercise prescription. Forty-two per cent of the players had an average in-season weekly compliance higher than our recommendations, two players (1\%) did not complete any of the recommended preseason training sessions and seven players (3\%) did not complete any of the recommended in-season training sessions. Players included in the PP analyses completed $93 \%$ of the recommended preseason training sessions and on average completed the programme 0.9 times per week (range $0.7-1.0$ ) in-season.

\section{Adverse effects}

We had no reports on any adverse events related to performing the study intervention.

\section{DISCUSSION}

This is the first study investigating the effect of an Adductor Strengthening Programme using a single-exercise approach to reduce the prevalence of groin problems in football players. Our

Table 5 Characteristics of the groin problems reported during the 28 weeks of the competitive season for players included in the intention-to-treat analysis

\begin{tabular}{lll}
\hline & $\begin{array}{l}\text { Intervention group } \\
(\mathbf{n}=\mathbf{8 4 4} \text { groin problems) }\end{array}$ & $\begin{array}{l}\text { Control group } \\
(\mathrm{n}=1321 \text { groin problems) }\end{array}$ \\
\hline Time loss (\%) & 41 & 33 \\
Gradual onset (\%) & 78 & 79 \\
Acute onset (\%) & 22 & 21 \\
Dominant leg (\%) & 45 & 44 \\
Non-dominant leg (\%) & 35 & 34 \\
Both legs (\%) & 20 & 22 \\
\hline
\end{tabular}

main finding was that the Adductor Strengthening Programme reduced the prevalence of groin problems among male football players; the risk of reporting groin problems during the competitive season was $41 \%$ lower, in favour of the intervention group in the ITT analysis and $47 \%$ in the PP analysis.

Our Adductor Strengthening Programme is the first groin-specific exercise-based programme to demonstrate efficacy in reducing the risk of groin injuries in male footballers. The hip adductors were specifically targeted in this programme, while previous studies have had a combined focus on hip adductors, abdominals and flexors. ${ }^{20}{ }^{2728}$ Furthermore, the intensive protocol implemented during the preseason is considered vital to gain effect of the programme prior to the injury counting during the regular season. This is in contrast to previous studies, which have implemented the prevention programme and injury registration at the same time..$^{27} 28$

Another reason why we may have found an effect where previous studies have not is that the compliance reported in the present study was much higher than what is often seen in sports injury prevention trials. ${ }^{329-31}$ On average, the players completed approximately $70 \%$ of the recommended protocol during both preseason and the competitive season. Actually, $42 \%$ of the players included in the ITT analysis had an average in-season weekly compliance higher than our recommendations. However, compliance was self-reported by the players and we do not know if they performed the exercise as often as reported. The compliance reported in other studies examining the preventive effect of an exercise programme on groin injury rate varies. Engebretsen et al reported that players had a 19\% compliance with a programme including static and dynamic hip adduction strength and abdominal exercises, ${ }^{27}$ while studies examining the effect of the FIFA 11 have reported from $52 \%$ to $73 \%$ compliance with the programme. ${ }^{30}{ }^{31}$ Silvers-Granelli et al reported a mean utilisation of the FIFA $11+$ of 30 sessions during a season, which they interpreted as moderate. ${ }^{5}$ Hölmich et al did not report any data on compliance with their programme including static and dynamic hip adduction strength, abdominal exercises and stretching. ${ }^{28}$ Compliance is believed to be a key factor. In two large RCTs on the FIFA 11+ programme, the risk of sustaining an injury was lower for the high compliance group, compared with players having intermediate or low compliance. ${ }^{56}$ This is also indicated in our analysis, where the PP analysis showed an even lower risk of groin problems than the ITT analysis.

The single-exercise approach in the present study must be considered a large advantage as the time required by the teams is short. The in-season protocol of the Adductor Strengthening Programme consists of only 1 weekly session, which takes $<5$ min to perform. However, this may also be considered a limitation as we have only specifically targeted one of four defined clinical entities for groin pain. ${ }^{32}$ We would argue that targeting the adductors addresses the main problem as adductor-related groin pain accounts for $>2 / 3$ of all hip and groin injuries in football. ${ }^{211-14}$ It is unknown specifically which entities were affected by the Adductor Strengthening Programme.

The effect of the exercise prescription dosage on hip adduction strength is unknown. We have data on different protocols of the CA, as used at level 3 in the current programme. A $36 \%$ increase in eccentric hip adduction strength was found in players performing the CA intensively for 8 weeks, ${ }^{19}$ whereas a less intensive 8-week protocol used in a different study showed an $8 \%$ increase. ${ }^{18}$ The preseason protocol used in the present study, with only one set per side per session, is likely to induce a strength increase in the lower range of these results. Additionally, we did not register the level of the protocol performed by each 
player. As previous studies indicate a dose-response relationship on eccentric adduction strength, ${ }^{18} 19$ it can be speculated that a higher intensity protocol would result in an even lower risk of groin problems. The optimal exercise prescription to maximise the effects on injury risk needs, however, further exploration.

Although we had no reports of any adverse effects, the lack of a systematic approach to register events should be considered a limitation of the study. However, our findings are similar to other studies showing no reports of any adverse effects when performing the CA. ${ }^{18} 19$ Furthermore, players in these studies reported very low delayed-onset muscle soreness (DOMS) in the hip adductors when performing the exercise. The individual highest values were related to the starting date, increase in load or associated with football training. ${ }^{18} 19$ Thus, we think that the Adductor Strengthening Programme is safe to perform and the careful progression in the exercise prescription was important to reduce the risk of DOMS and adverse events to a minimum.

The preventive effect from structured interventions like the FIFA $11+$ is well known; ${ }^{4}$ however, the specific effects of these general programmes are unclear. Only two papers report effects on hip/groin injuries, with conflicting results. ${ }^{56}$ In the same way that the Nordic Hamstring exercise is included in the FIFA $11+$ to specifically target hamstring injuries, the three progression levels of the Adductor Strengthening Programme are suggested to be included in the FIFA 11+ programme to specifically target the adductor muscles and their associated groin problems most often seen in male football.

\section{Methodological considerations}

A strength of this trial is the use of the OSTRC Overuse Injury Questionnaire. This method has been shown to be more sensitive than injury recording based on a time-loss definition, capturing all groin problems. ${ }^{733}$ Actually, a promising non-significant $31 \%$ reduction in groin injury rates was shown in 977 Danish amateur male football players. ${ }^{28}$ The results from this trial indicate that the study has been underpowered to detect an effect on time-loss injuries. Our data therefore represent a more complete picture of the extent of groin problems in football, with a large share of problems leading to reduced training participation, training volume or performance but fewer leading to time loss.

Another strength of this trial is the inclusion of players already having groin problems. Traditionally, players with problems at baseline are excluded, recording only new cases during the intervention period. From a previous study, we know that $>50 \%$ of the players reporting groin pain in the previous season still had pain at the beginning of the new season. ${ }^{9}$ Removing players having groin symptoms at baseline would have resulted in a group not being representative for the population of footballers where groin problems are very common.

There are some methodological limitations in this study. First, the retrospective registration during the last month of the study may induce a recollection bias and represents a limitation to the injury registration. The decision to register retrospectively groin problems was done to ensure sufficient data for the analyses. Aiming to reduce recall bias, players had access to a document summarising their previous questionnaire responses and match fixtures when completing the retrospective registration. Still, there is a potential for recall bias; however, it seems highly unlikely that this would affect players in the intervention and control groups differently.

Second, deviations from the registered protocol should be considered a limitation of any trial. The decision to remove players with a low response rate was done to ensure sufficient data to perform imputation. The majority (77\%) of the enrolled players were included in the GEE analyses. Although the postrandomised change of criteria for the analyses may introduce bias, ${ }^{34}$ the decision to diverge from the protocol was taken prior to the analyses and was based on the response rate, irrespective of whether or not they had actually performed the Adductor Strengthening Programme. In addition, difference in subscales scores of the HAGOS between the players removed from the analysis, compared with the once included, indicates that players with lower response rate $(<75 \%)$ had more severe symptoms at baseline. However, we considered these small differences not clinically relevant. Furthermore, there were no differences in any of the subscales when comparing removed players from the intervention group with the ones removed from the control group.

Third, a limitation of the injury registration method used in the present study is the lack of detailed diagnostic information on each case. Reliable medical follow-up was not feasible given the scale of the study. Instead we attempted to obtain diagnostic information by inviting players reported to have a groin problem affecting match play for more than two consecutive weeks to a standardised examination. However, as most of the teams already had their own medical teams, only 26 players contacted us and were examined. In a future study, the self-reported groin problems should be examined, classified and reported according to the Doha agreement on terminology and definitions. ${ }^{32}$

\section{Perspectives}

The results from the present study suggest that the Adductor Strengthening Programme should be included in football training, among senior male football players. Whether the preventive effect from the Adductor Strengthening Programme can be generalised to female or youth-level football players, as well players at the highest professional level, is not known. Other types of athletes may also benefit from the programme as low hip adduction strength is also considered a risk factor associated with groin problems in other sports with similar movement patterns, such as ice hockey, rugby and Australian rule football. ${ }^{35-38}$

\section{CONCLUSION}

The Adductor Strengthening Programme substantially reduced the prevalence and risk of groin problems in male football players. We recommend that the programme be implemented as a part of normal football training.

What are the findings?

- The Adductor Strengthening Programme, based on one single exercise with different progression levels, reduced the prevalence and risk of groin problems in male football players by $41 \%$.

How might it impact on clinical practice in the future?

- The Adductor Strengthening Programme should be implemented as a part of normal football training.

- We suggest to include the three progression levels of the Adductor Strengthening Programme in the FIFA $11+$ programme to specifically target the adductor muscles. 
Twitter Follow @jharoey, @benclarsen, @aserner, @kthorborg, @perholmich, @ docthorandersen, @roaldbahr, @ostrc1.

Acknowledgements The authors thank EE Eriksen, M Lislevand, K Nordanger Martin, K Hammond Rosbach, A Kjøsnes, A Megård and ID Andersen for their assistance in data collection. They also thank all players and coaches who participated in the study, as well as the team medical staff.

Contributors With exception of EGW and MG $\varnothing$, all authors have been involved in the planning of the study. JH, BC, EGW, MGØ, TEA and RB performed the data collection. All authors have been involved in the data analyses, drafting and revision of the manuscript, and have approved the final version.

Funding The Oslo Sports Trauma Research Center has been established at the Norwegian School of Sport Sciences through generous grants from the Royal Norwegian Ministry of Culture, the South-Eastern Norway Regional Health Authority, the International Olympic Committee, the Norwegian Olympic Committee \& Confederation of Sport, and Norsk Tipping AS.

Competing interests None declared.

Patient consent Not required.

Ethics approval This cluster-randomised controlled trial was registered with the International Standard Randomised Controlled Trial Number registry (ISRCTN98514933). The study was approved by the South-Eastern Norway Regional Committee for Medical Research Ethics (2015/1922/REK) and the Norwegian Data Inspectorate (45388/3/LT/LR).

Provenance and peer review Not commissioned; externally peer reviewed.

Data sharing statement The players were not involved in the design of research question, outcome measures, design or recruitment to the study. The results from the study will be disseminated to all teams that were included in the project. Applications for data sharing can be made.

(c) Article author(s) (or their employer(s) unless otherwise stated in the text of the article) 2019. All rights reserved. No commercial use is permitted unless otherwise expressly granted.

\section{REFERENCES}

1 Waldén $M$, Hägglund $M$, Ekstrand J. The epidemiology of groin injury in senior football: a systematic review of prospective studies. Br J Sports Med 2015;49:792-7.

2 Werner J, Hägglund $M$, Waldén $M$, et al. UEFA injury study: a prospective study of hip and groin injuries in professional football over seven consecutive seasons. Br J Sports Med 2009;43:1036-40.

3 Esteve E, Rathleff MS, Bagur-Calafat C, et al. Prevention of groin injuries in sports: a systematic review with meta-analysis of randomised controlled trials. Br I Sports Med 2015;49:785-91.

4 Thorborg K, Krommes KK, Esteve E, et al. Effect of specific exercise-based football injury prevention programmes on the overall injury rate in football: a systematic review and meta-analysis of the FIFA 11 and 11+ programmes. Br J Sports Med 2017:51:562-71.

5 Silvers-Granelli H, Mandelbaum B, Adeniji 0, et al. Efficacy of the FIFA 11+ Injury prevention program in the collegiate male soccer player. Am J Sports Med 2015;43:2628-37.

6 Soligard T, Myklebust G, Steffen K, et al. Comprehensive warm-up programme to prevent injuries in young female footballers: cluster randomised controlled trial. BMJ 2008;337:a2469.

7 Harøy J, Clarsen B, Thorborg K, et al. Groin Problems in Male Soccer Players Are More Common Than Previously Reported. Am J Sports Med 2017;45:1304-8.

8 Thorborg K, Branci S, Nielsen MP, et al. Copenhagen five-second squeeze: a valid indicator of sports-related hip and groin function. Br J Sports Med 2017;51:594-9.

9 Thorborg K, Rathleff MS, Petersen P, et al. Prevalence and severity of hip and groin pain in sub-elite male football: a cross-sectional cohort study of 695 players. Scand J Med Sci Sports 2017:27.

10 Clarsen B, Myklebust G, Bahr R. Development and validation of a new method for the registration of overuse injuries in sports injury epidemiology: the Oslo Sports Trauma Research Centre (OSTRC) overuse injury questionnaire. Br I Sports Med 2013;47:495-502.

11 Hölmich P, Thorborg K, Dehlendorff C, et al. Incidence and clinical presentation of groin injuries in sub-elite male soccer. Br J Sports Med 2014;48:1245-50.

12 Mosler $A B$, Weir $A$, Eirale $C$, et al. Epidemiology of time loss groin injuries in a men's professional football league: a 2-year prospective study of 17 clubs and 606 players. Br J Sports Med 2018;52.

13 Serner A, Tol JL, Jomaah N, et al. Diagnosis of acute groin injuries: a prospective study of 110 athletes. Am J Sports Med 2015;43:1857-64.
14 Taylor R, Vuckovic Z, Mosler A, et al. Multidisciplinary Assessment of 100 Athletes With Groin Pain Using the Doha Agreement: High Prevalence of Adductor-Related Groin Pain in Conjunction With Multiple Causes. Clin I Sport Med 2017.

15 Engebretsen AH, Myklebust G, Holme I, et al. Intrinsic risk factors for groin injuries among male soccer players: a prospective cohort study. Am I Sports Med 2010;38:2051-7.

16 Whittaker JL, Small C, Maffey L, et al. Risk factors for groin injury in sport: an updated systematic review. Br J Sports Med 2015;49:803-9.

17 Thorborg K, Branci S, Nielsen MP, et al. Eccentric and isometric hip adduction strength in male soccer players with and without adductor-related groin pain: an assessorblinded comparison. Orthop J Sports Med 2014;2:232596711452177.

18 Harøy J, Thorborg K, Serner A, et al. Including the Copenhagen Adduction Exercise in the FIFA 11+ Provides Missing Eccentric Hip Adduction Strength Effect in Male Soccer Players: a randomized controlled trial. Am I Sports Med 2017;45:3052-9.

19 Ishøi L, Sørensen CN, Kaae NM, et al. Large eccentric strength increase using the Copenhagen Adduction exercise in football: A randomized controlled trial. Scand J Med Sci Sports 2016;26.

20 Krommes K, Bandholm T, Jakobsen MD, et al. Dynamic hip adduction, abduction and abdominal exercises from the holmich groin-injury prevention program are intense enough to be considered strengthening exercises - a cross-sectional study. Int I Sports Phys Ther 2017;12:371-80.

21 Serner A, Jakobsen MD, Andersen LL, et al. EMG evaluation of hip adduction exercises for soccer players: implications for exercise selection in prevention and treatment of groin injuries. Br J Sports Med 2014;48:1108-14.

22 Campbell MK, Piaggio G, Elbourne DR, et al. Consort 2010 statement: extension to cluster randomised trials. BMJ 2012;345:e5661.

23 Hoffmann TC, Glasziou PP, Boutron I, et al. Better reporting of interventions: template for intervention description and replication (TIDieR) checklist and guide. BMJ 2014;348:g1687.

24 Thorborg K, Hölmich P, Christensen R, et al. The Copenhagen Hip and Groin Outcome Score (HAGOS): development and validation according to the COSMIN checklist. Br J Sports Med 2011;45:478-91.

25 Hawker GA, Mian S, Kendzerska T, et al. Measures of adult pain: Visual Analog Scale for Pain (VAS Pain), Numeric Rating Scale for Pain (NRS Pain), McGill Pain Questionnaire (MPQ), Short-Form McGill Pain Questionnaire (SF-MPQ), Chronic Pain Grade Scale (CPGS), Short Form-36 Bodily Pain Scale (SF-36 BPS), and Measure of Intermittent and Constant Osteoarthritis Pain (ICOAP). Arthritis Care Res 2011;63(Suppl 11):S240-52.

26 van Buuren S. Flexible Imputation of Missing Data. Hoboken: Taylor and Francis, 2012.

27 Engebretsen AH, Myklebust G, Holme I, et al. Prevention of injuries among male soccer players: a prospective, randomized intervention study targeting players with previous injuries or reduced function. Am I Sports Med 2008:36:1052-60.

28 Hölmich P, Larsen K, Krogsgaard K, et al. Exercise program for prevention of groin pain in football players: a cluster-randomized trial. Scand J Med Sci Sports 2010;20:814-21.

29 Goode AP, Reiman MP, Harris L, et al. Eccentric training for prevention of hamstring injuries may depend on intervention compliance: a systematic review and metaanalysis. Br J Sports Med 2015;49:349-56.

30 Steffen K, Myklebust G, Olsen OE, et al. Preventing injuries in female youth football--a cluster-randomized controlled trial. Scand I Med Sci Sports 2008;18:605-14.

31 van Beijsterveldt AM, van de Port IG, Krist MR, et al. Effectiveness of an injury prevention programme for adult male amateur soccer players: a cluster-randomised controlled trial. Br J Sports Med 2012;46:1114-8.

32 Weir A, Brukner P, Delahunt $E$, et al. Doha agreement meeting on terminology and definitions in groin pain in athletes. Br J Sports Med 2015;49:768-74.

33 Clarsen B, Bahr R, Heymans MW, et al. The prevalence and impact of overuse injuries in five Norwegian sports: Application of a new surveillance method. Scand J Med Sci Sports 2015;25.

34 Nüesch $\mathrm{E}$, Trelle $\mathrm{S}$, Reichenbach $\mathrm{S}$, et al. The effects of excluding patients from the analysis in randomised controlled trials: meta-epidemiological study. $B M J$ 2009;339:b3244.

35 Crow JF, Pearce AJ, Veale JP, et al. Hip adductor muscle strength is reduced preceding and during the onset of groin pain in elite junior Australian football players. I Sci Med Sport 2010;13:202-4.

36 Emery CA, Meeuwisse WH. Risk factors for groin injuries in hockey. Med Sci Sports Exerc 2001;33:1423-33.

$37 \mathrm{O}^{\prime}$ Connor D. Groin injuries in professional rugby league players: a prospective study. J Sports Sci 2004;22:629-36.

38 Tyler TF, Nicholas SJ, Campbell RJ, et al. The association of hip strength and flexibility with the incidence of adductor muscle strains in professional ice hockey players. Am J Sports Med 2001;29:124-8. 